\title{
Pharmacological Stimulation of Type 5 Adenylyl Cyclase Stabilizes Heart Rate Under Both Microgravity and Hypergravity Induced by Parabolic Flight
}

\author{
Yunzhe Bai ${ }^{1}$, Takashi Tsunematsu ${ }^{1}$, Qibin Jiao ${ }^{1}$, Yoshiki Ohnuki ${ }^{2}$, Yasumasa Mototani ${ }^{2}$, \\ Kouichi Shiozawa ${ }^{2}$, Meihua Jin ${ }^{1}$, Wenqian $\mathrm{Cai}^{1}$, Hui-Ling Jin ${ }^{1}$, Takayuki Fujita ${ }^{1}$, Yasuhiro Ichikawa ${ }^{1}$, \\ Kenji Suita ${ }^{1}$, Reiko Kurotani ${ }^{1,3}$, Utako Yokoyama ${ }^{1}$, Motohiko Sato ${ }^{1}$, Kousaku Iwatsubo ${ }^{4}$, \\ Yoshihiro Ishikawa ${ }^{1,4}$, and Satoshi Okumura ${ }^{1,2, *}$ \\ ${ }^{1}$ Cardiovascular Research Institute, Yokohama City University Graduate School of Medicine, Yokohama 236-0004, Japan \\ ${ }^{2}$ Department of Physiology, Tsurumi University School of Dental Medicine, Yokohama 230-8501, Japan \\ ${ }^{3}$ Biochemical Engineering, Faculty of Engineering, Yamagata University, Yonezawa, Yamagata 992-8501, Japan \\ ${ }^{4}$ Cardiovascular Research Institute, Department of Cell Biology \& Molecular Medicine and Medicine (Cardiology), \\ New Jersey Medical School of UMDNJ, Newark, New Jersey 07103, USA
}

Received April 25, 2012; Accepted June 21, 2012

\begin{abstract}
We previously demonstrated that type 5 adenylyl cyclase (AC5) functions in autonomic regulation in the heart. Based on that work, we hypothesized that pharmacological modulation of AC5 activity could regulate the autonomic control of the heart rate under micro- and hypergravity. To test this hypothesis, we selected the approach of activating AC5 activity in mice with a selective AC5 activator (NKH477) or inhibitor (vidarabine) and examining heart rate variability during parabolic flight. The standard deviation of normal R-R intervals, a marker of total autonomic variability, was significantly greater under micro- and hypergravity in the vidarabine group, while there were no significant changes in the NKH477 group, suggesting that autonomic regulation was unstable in the vidarabine group. The ratio of low frequency and high frequency (HF) in heart rate variability analysis, a marker of sympathetic activity, became significantly decreased under micro- and hypergravity in the NKH477 group, while there was no such decrease in the vidarabine group. Normalized HF, a marker of parasympathetic activity, became significantly greater under micro- and hypergravity in the NKH477 group. In contrast, there was no such increase in the vidarabine group. This study is the first to indicate that pharmacological modulation of AC5 activity under micro- and hypergravity could be useful to regulate the autonomic control of the heart rate.
\end{abstract}

Keywords: adenylyl cyclase isoform, autonomic nerve activity, parabolic flight, heart rate variability, microgravity

\section{Introduction}

Cardiac function is regulated by the autonomic nervous system. Sympathetic regulation leads to coupling of the $\beta$-adrenergic receptor ( $\beta$-AR) and Gs, the G-protein responsible for stimulating activity of adenylyl cyclase (AC), a membrane-bound enzyme that catalyzes the

*Corresponding author. okumura-s@tsurumi-u.ac.jp Published online in J-STAGE on July 31, 2012 (in advance) doi: 10.1254 /jphs.12102FP conversion of ATP to cyclic AMP (cAMP), thereby stimulating protein kinase A (PKA) and ultimately increasing cardiac contractility and heart rate (HR) $(1-5)$. Parasympathetic regulation counteracts these effects through the activation of the muscarinic receptor and Gi, the G-protein that can inhibit cardiac contractility and heart rate (6). The autonomic nervous system constitutes the two arms of regulation in the heart. We developed a mouse model with disruption of a major AC isoform (type 5) in the heart and demonstrated that type $5 \mathrm{AC}$ (AC5) regulates cardiac function through the parasympa- 
thetic arm of the autonomic nervous system, as well as through the sympathetic arm $(7-9)$.

The autonomic nervous system is known to be altered under microgravity (10) and hypergravity $(11,12)$. We have previously examined the role of AC5 in the regulation of the autonomic nervous system in the heart under micro- and hypergravity induced by parabolic flights using transgenic mouse models with AC5 overexpressed in the heart (AC5TG) or with disrupted AC5 (AC5KO) and analyzed heart rate variability during parabolic flight. Changes in heart rate variability (HRV) in response to micro- and hypergravity were augmented in AC5KO, but attenuated in AC5TG, suggesting that AC5 plays an important role in stabilizing heart rate control via autonomic regulation under gravitational stress (13).

We hypothesized that pharmacological regulation of AC5 activity could modulate the autonomic control of the heart rate under gravitational stress during parabolic flight. To test this hypothesis, we decided to examine the effect on HRV of activating or inhibiting AC5 activity in wild-type mice with a selective AC5 activator: 6-[3(dimethylamino)propionyl]forskolin (NKH477) (14) or an AC5 inhibitor: vidarabine (15) during parabolic flight. We found that HR was stabilized by increasing AC5 activity with NKH477, but destabilized by decreasing AC5 activity with vidarabine under micro- and hypergravity during parabolic flight, in accordance with our previous findings using AC5TG and AC5KO.

We also examined the the ratio between low frequency (LF) and high frequency (HF), a marker of sympathetic activity, and normalized HF (nHF), a marker of parasympathetic activity during the parabolic flight and found that AC5 activity plays an important role to show continuous responses in these autonomic parameters such as LF/HF or nHF, under micro- and hypergravity (16).

Many astronauts suffer to varying degrees from symptoms suggestive of autonomic dysfunction such as nausea, vomiting, and dizziness during the early days of space flight (17), as well as showing severe orthostatic intolerance for several days after spaceflight $(18,19)$. The underlying molecular mechanism and appropriate treatment for the autonomic dysfunction in space and on earth after space flight remain unknown. However, not only trained astronauts but also normal subjects will stay in space in the near future. Thus, it is important to clarify the mechanism and to establish suitable treatment of autonomic dysfunction during and after spaceflight. This study is the first to demonstrate that pharmacological activation/inhibition of AC5 activity can regulate the autonomic control of the heart rate under micro- and hypergravity, indicating the importance of regulating AC5 activity for the treatment of autonomic dysfunction in space and on earth after spaceflight.

\section{Materials and Methods}

\section{Parabolic flight experiment}

Parabolic flight experiments were performed in a jet airplane operated by the Diamond Air Service in Nagoya using the same protocol previously employed by us (13). Briefly, in one parabolic flight, which lasted for approximately $1 \mathrm{~h}, 8-12$ parabolas were performed in total with a 4-6-min interval between consecutive parabolas. At the end of each parabolic flight, mice were euthanized by cervical dislocation and replaced with the next mice. Parabolic flight was divided into five phases (Fig. 1). The first phase (I) was in normogravity (1 G, G: gravity), the second phase (II) was in hypergravity $(1.3 \mathrm{G})$, the third phase (III) was in microgravity $(0.03 \mathrm{G})$, the fourth phase (IV) was in hypergravity $(1.8 \mathrm{G})$, and the fifth phase $(\mathrm{V})$ was in normogravity $(1 \mathrm{G})$. Each parabola provided $15-20 \mathrm{~s}$ of microgravity (phase III) and hypergravity (phase IV) and we evaluated data from the first parabola, except in Figs. 3B, 4B, 5B, 6B, in which the $\mathrm{G}$ force in the hypergravity phase (phase II) before the microgravity phase was kept below $1.3 \mathrm{G}$ to avoid excessive gravity stress on mice and the effect of previous parabolas. During the experiment, the temperature in the plane was kept at $22^{\circ} \mathrm{C} \pm 2{ }^{\circ} \mathrm{C}$ and air pressure was $0.9 \pm 0.1$ atm.

\section{Animals}

Male C57BL/6CrSlc mice aged 4 months were used for this experiment (Japan SLC, Inc., Hamamatsu). The

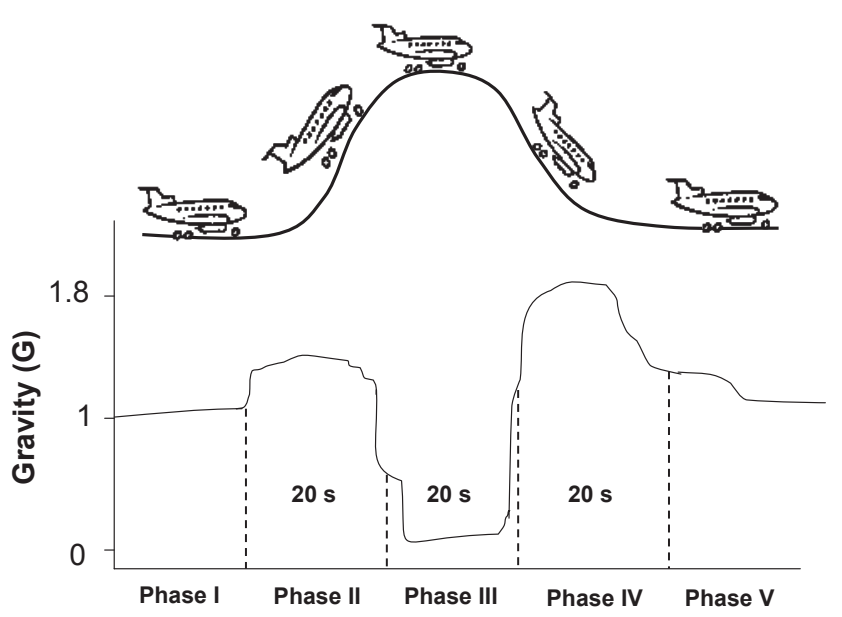

Fig. 1. Schematic representation of parabolic flight. Each parabola provided approximately $20 \mathrm{~s}$ of $0.03 \mathrm{G}$ (G; gravity). Parabolic flight was divided into five phases. The first phase (I) was normogravity (1 $\mathrm{G})$, the second phase (II) was hypergravity $(1.3 \mathrm{G})$, the third phase (III) was microgravity $(0.03 \mathrm{G})$, the fourth phase (IV) was hypergravity $(1.8 \mathrm{G})$, and the fifth phase $(\mathrm{V})$ was normogravity $(1 \mathrm{G})$. The Y-axis indicates changes in gravity. 
care and treatment of the animals were carried out according to the Japanese Government Animal Protection and Management Law (no. 105) and the Guidelines for Animal Experiments of Yokohama City University School of Medicine.

\section{Experimental design and protocol}

ECG recordings were obtained with a telemetric unit (PhysioTel; Data Sciences International, St. Paul, MN, USA). Infusion of NKH477 (1 mg kg $\left.{ }^{-1} \cdot \mathrm{day}^{-1}\right)(14)$, vidarabine $\left(15 \mathrm{mg} \cdot \mathrm{kg}^{-1} \cdot \mathrm{day}^{-1}\right)$ (15), or phosphate-buffered saline (PBS) as a control was done via an osmotic minipump (Model 2001; ALZET, Cupertino, CA, USA), which was implanted $16 \mathrm{~h}$ before the parabolic flight.

\section{Time-domain measures}

Conscious mice were separately placed in doublewalled plastic cages, which were placed on a receiver in a special rack within the aircraft, and data from the freely moving mice were recorded by the data acquisition system during flight, as described previously by us (13). HRV measurements included mean HR, R-R interval (mean, max, minimum) and the standard deviation of normal R-R intervals (SDNN). The different G phases causes important changes in the R-R interval. Therefore, we also used the coefficient of variation $(\mathrm{CV}, \%)$ as a normalized index of SDNN (CV-SDNN). SDNN $(\mathrm{ms})=\mathrm{SD}$ of all normal R-R intervals; CV-SDNN $(\%)=\mathrm{SDNN} /$ mean $\mathrm{R}-\mathrm{R} \times 100$.

\section{Frequency-domain measures}

In the frequency domain, the power spectral density was calculated by applying the fast Fourier transformation (FFT) to overlapping segments of the resampled data and by averaging the spectral results $(16,20)$. The FFT was calculated by using 512 points and half overlap with a Hann window. Cutoff frequencies divided the power spectrum into two main parts, LF $(0.4-1.5 \mathrm{~Hz})$ and $\mathrm{HF}(1.5-4.0 \mathrm{~Hz})$ powers and were determined by multiplying the standard frequencies used in human studies by 10 to account for HR differences between mice and humans, as recommended by Gehrmann et al. (21). HF powers were normalized to account for differences in total power (TP) between animals by multiplying the power region of interest by 100 and dividing by the difference between TP and very LF power $(0.0-0.4 \mathrm{~Hz})$ (16).

\section{Statistical analyses}

All data are reported as the mean \pm S.E.M. Statistical comparisons were performed with the Kruskal-Wallis nonparametric test followed by the Dunn test (Figs. 2, 3, 7, 8). Differences were considered significant at $P<0.05$.

\section{Results}

\section{Effect of parabolic flight on HR}

Mean HR in the NKH477, vidarabine, and control groups were evaluated in each phase of parabolic flight during the first parabola. The HR in phase $I$ in the NKH477 group was significantly higher than that in the control group [control group $(\mathrm{n}=12): 558 \pm 81$ vs. NKH477 group $(\mathrm{n}=8)$ : $681 \pm 38$ bpm; $P<0.01$ ] (Fig.
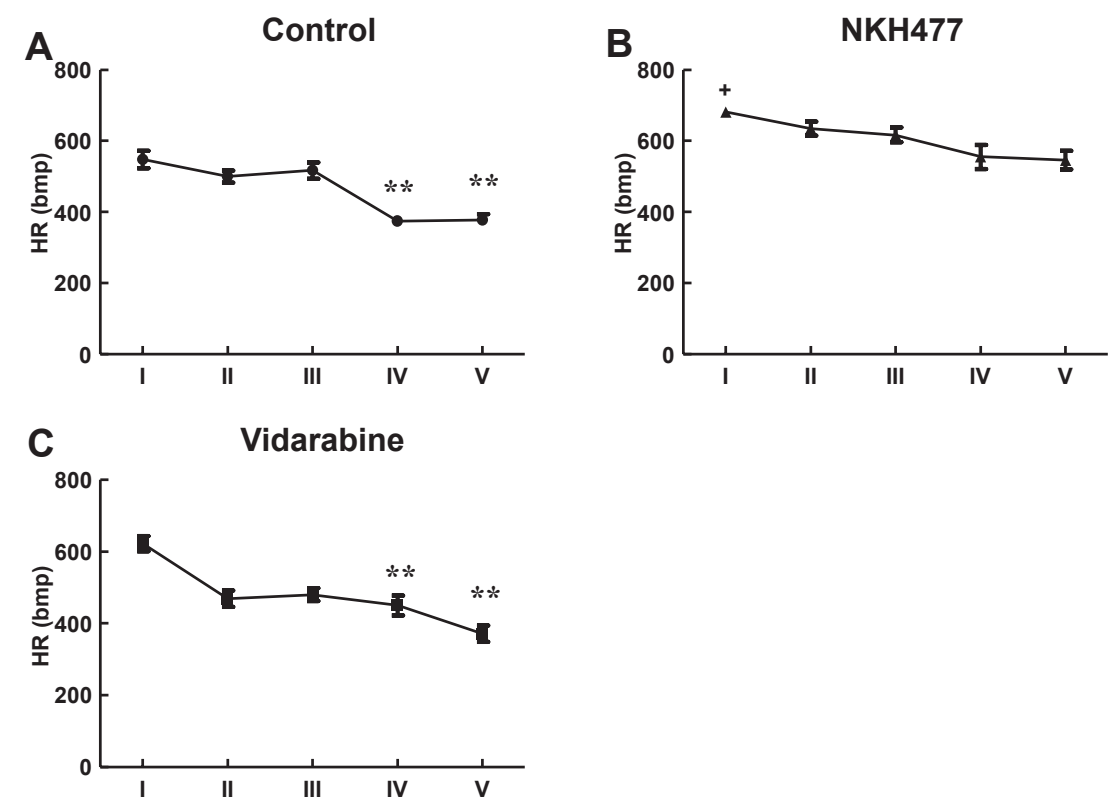

Fig. 2. Comparison of HR during parabolic flights. Comparison of HR among phases I, II, III, $\mathrm{IV}$, and $\mathrm{V}$ in the control $(\mathrm{n}=12)(\mathrm{A}), \mathrm{NKH} 477$ $(\mathrm{n}=8)(\mathrm{B})$, vidarabine $(\mathrm{n}=8)(\mathrm{C})$ groups during the first parabola. HR was compared between phase I and phase II, III, IV, or V in each group $(* * P<0.01)$ and also between the control and NKH477 groups $\left({ }^{+} P<0.05\right)$. 
2B), as previously found in AC5TG $(13,22)$. HR showed a significant decrease in later phases (IV $-\mathrm{V}$ ) in the control and vidarabine groups, but there was no significant change in the NKH477 group, suggesting that HR was stabilized in the NKH477 group, compared with the other groups during parabolic flight.

\section{Effect of parabolic flight on $R-R$ interval of $H R V$}

Maximum, mean, and minimum R-R intervals in the three groups were examined during the first and eighth parabolas (Fig. 3). Maximum, mean, and minimum R-R intervals, in general, increased gradually in later phases (I through IV) during the first parabola (Fig. 3A). When maximum, mean, and minimum $\mathrm{R}-\mathrm{R}$ intervals were compared in each group during the first parabola, the degree of variability of the R-R interval was smaller in the NKH477 group $(n=8)$ and greater in the vidarabine group $(\mathrm{n}=8)$ than that in the control group $(\mathrm{n}=8)$. In the eighth parabola (Fig. 3B), the degree of variability remained unchanged in the vidarabine group $(n=8)$, suggesting that adaptations to micro- and hypergravity were impaired in the vidarabine group $(10,23)$.

For further analysis, the R-R intervals under normogravity (phase I), microgravity (phase III), and hypergravity (phase IV) were plotted by time (s) versus R-R (ms) interval in each group during the first (Figs. 4A, 5A, 6A) and eighth parabolas (Figs. 4B, 5B, 6B). The R-R intervals under normogravity (phase I) during the first parabola were stable in each group (Fig. 4A), but became more variable during the eighth parabola. However, the degree of variability was greater in the vidarabine group and smaller in the NKH477 group than that in the control group (Fig. 4B).

Under microgravity (phase III), the degree of variability was less in the NKH477 group, as compared with the other groups (Fig. 5A). However, the degree of variability was greater in the vidarabine group $(n=8)$ and smaller in the NKH477 group $(n=9)$ than that in the control group $(\mathrm{n}=8)$ during the eighth parabola (Fig. $5 B)$.

Under hypergravity (phase IV), the degree of variability showed a similar tendency to that under microgravity in the three groups during the first (Fig. 6A) and the eighth parabolas (Fig. 6B).

Taken together, these results may indicate that pharmacological activation of AC5 stabilizes the R-R intervals not only under microgravity but also under hypergravity during parabolic flight.

A
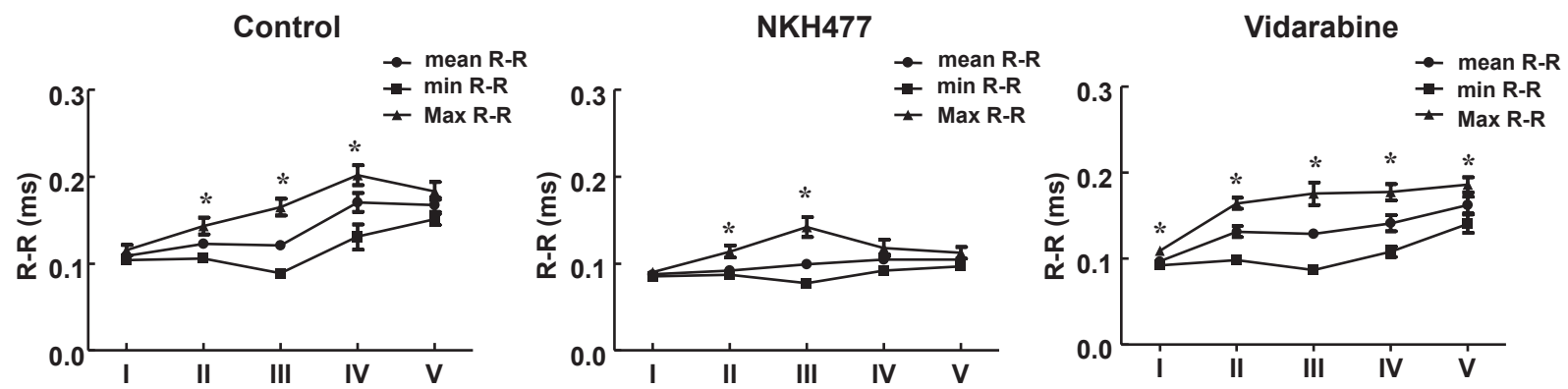

B
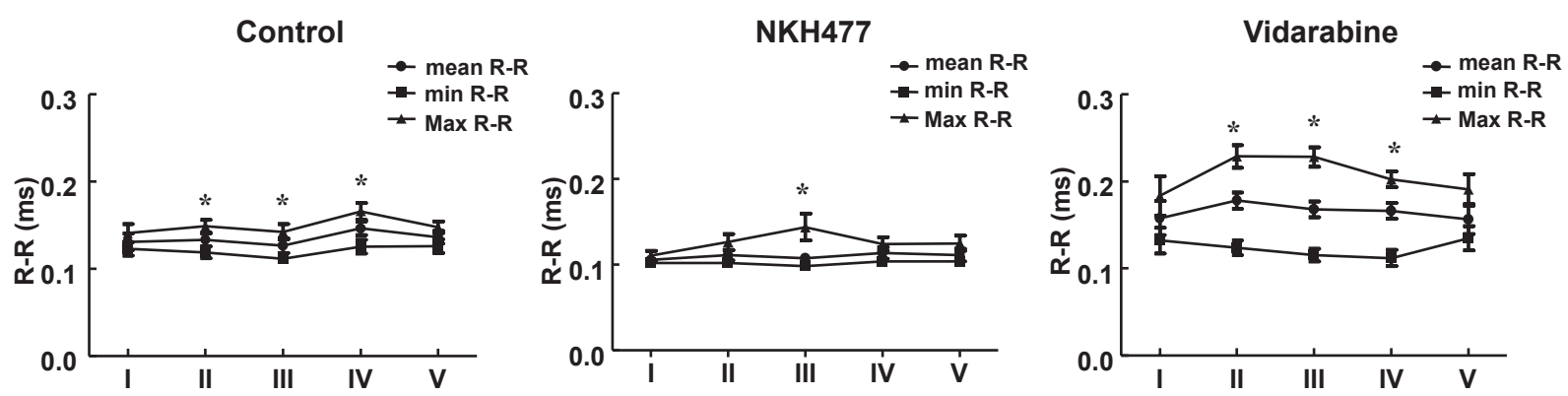

Fig. 3. Comparison of R-R interval during various phases of parabolic flights. Comparison of mean (mean), maximum (Max), and minimum ( $\mathrm{min})$ R-R intervals (ms) among phases I, II, III, IV, and V in the control ( $\mathrm{n}=8)$, NKH477 ( $=8-9)$, and vidarabine $(n=8)$ during the first parabola $(A)$ and eighth parabola $(B)$. Maximum R-R intervals were compared with the minimum R-R interval in each group $(* P<0.05)$. 


\section{Normogravity (Phase I)}

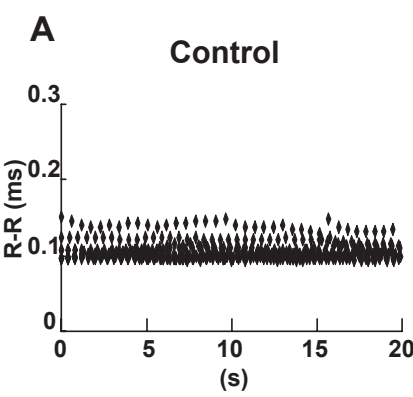

B

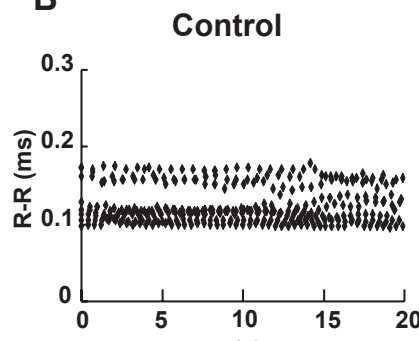

(s)

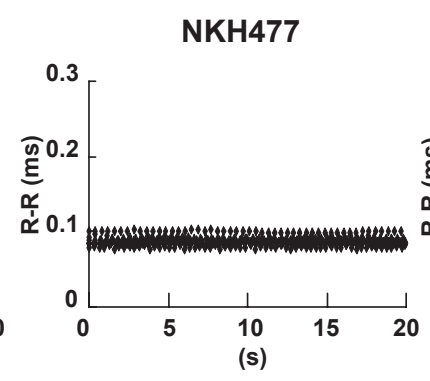

NKH477

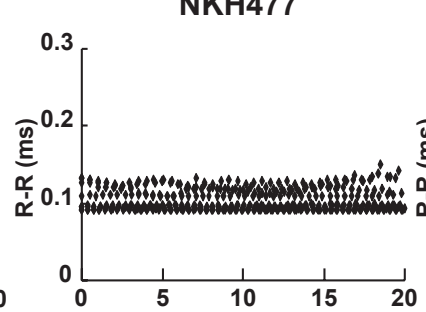

(s)

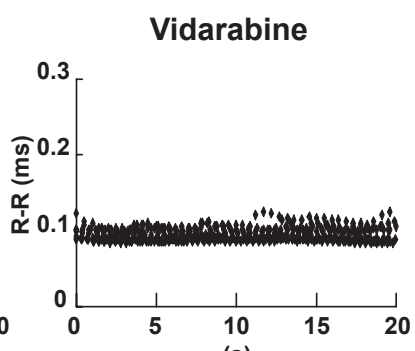

(s)

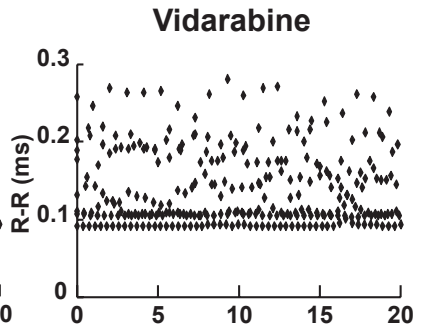

(s)

Fig. 4. Comparison of R-R interval under normogravity. R-R interval under normogravity (phase I) is shown by the plot of time (s) versus R-R interval $(\mathrm{ms})$ in the control $(\mathrm{n}=8)$, NKH477 ( $\mathrm{n}=8-9)$, vidarabine $(\mathrm{n}=$ 8) groups during the first (A) and eighth (B) parabolas.

A

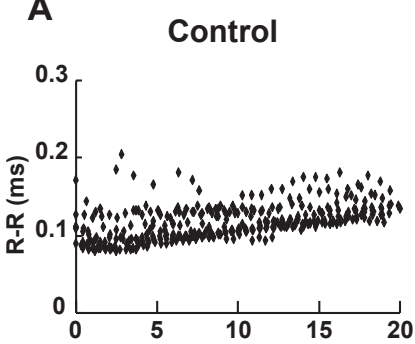

(s)

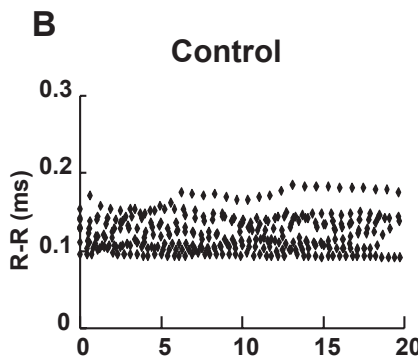

(s)

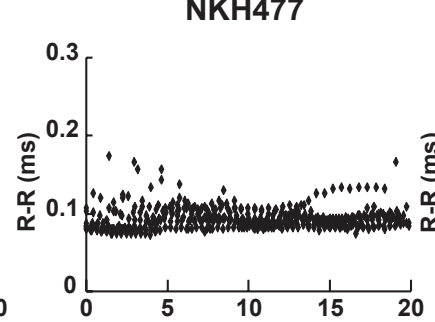

(s)

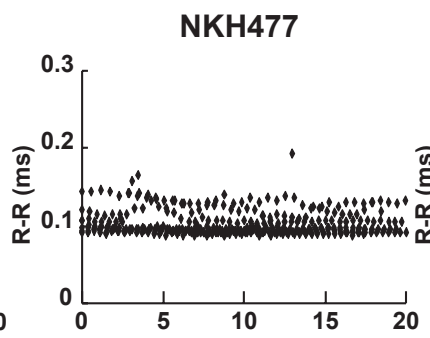

(s)

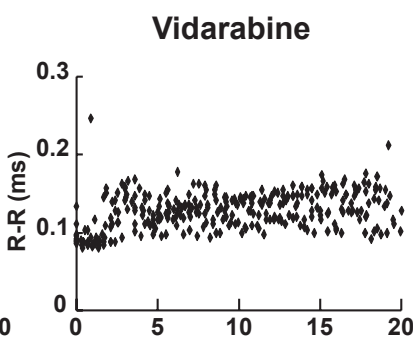

(s)

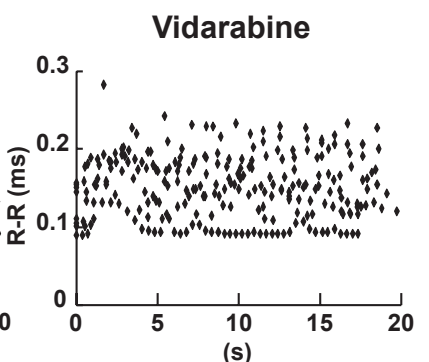

Fig. 5. Comparison of R-R interval under microgravity. Representative results under the microgravity (phase III) are shown by the plot of time (s) versus R-R interval ( $\mathrm{ms}$ ) in the control $(n=8)$, NKH477 $(n=8)$, and vidarabine $(n=8)$ groups during the first (A) and eighth (B) flights.

\section{Effect of parabolic flight on SDNN of HRV}

Because HR under gravitational stress is most likely regulated by the autonomic nervous system, we thus compared SDNN (Fig. 7A) and another parameter CVSDNN (Fig. 7B) as a measure of total autonomic instability (24). SDNN and CV-SDNN were both significantly greater under microgravity (phase III) and hypergravity (phase II and IV) than those under normogravity (phase I) in each group. Notably, the absolute value of SDNN under microgravity (phase III) as well as hypergravity (phase IV) was strikingly greater in the vidarabine group $(\mathrm{n}=8)$ and much smaller in the NKH477 group $(\mathrm{n}=9)$ than that in the control group $(n=12)$. CV-SDNN showed similar behavior, indicating that increased AC5 activity stabilizes the autonomic control of the heart rate under gravitational stress. 


\section{Hypergravity (Phase IV)}
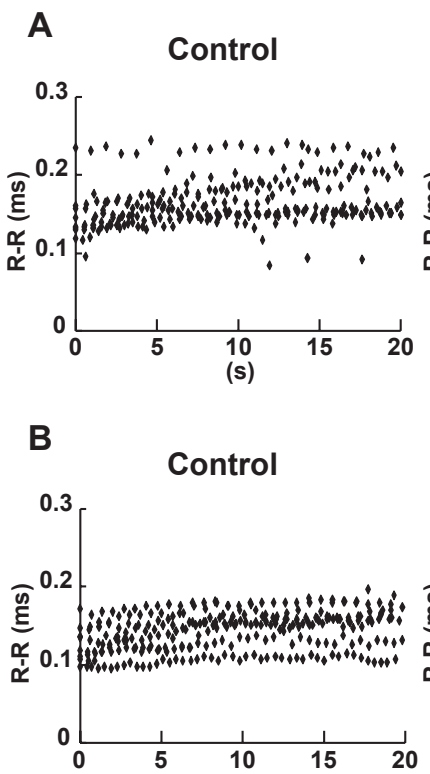

(s)

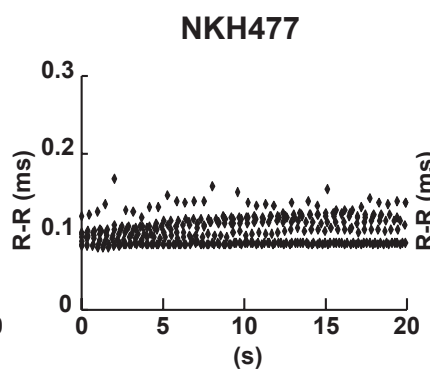

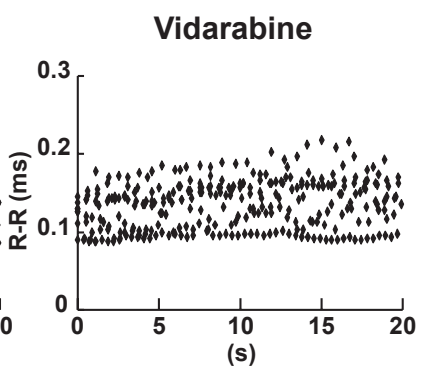

(s)
NKH477

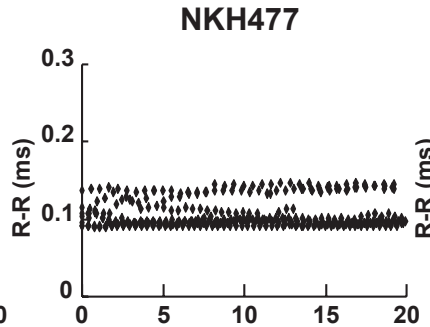

(s)

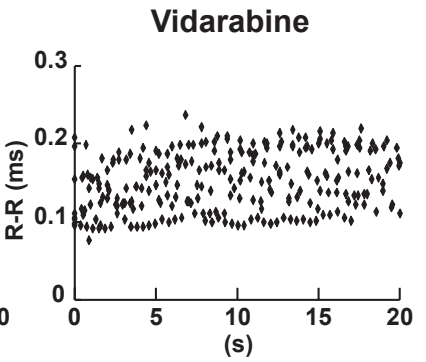

Fig. 6. Comparison of R-R interval under hypergravity. Representative results under the hypergravity (phase IV) are shown by the plot of time (s) versus R-R interval (ms) in the control $(\mathrm{n}=8)$, NKH477 $(\mathrm{n}=9)$, and vidarabine $(n=8)$ groups during the first (A) and eighth (B) flights.

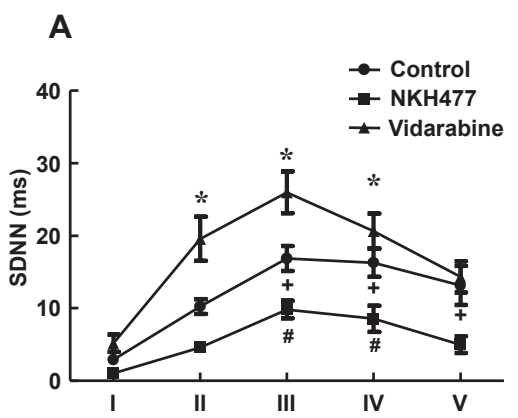

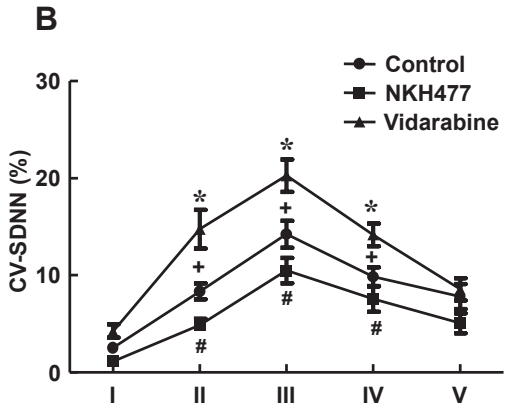

Fig. 7. Comparison of SDNN and CV-SDNN during parabolic flights. The standard deviation of normal R-R intervals (SDNN) (A) and coefficient of variation $(\mathrm{CV}, \%)$ as a normalized index of SDNN (CV-SDNN) (B) during the first parabola. These indexes were compared between phase I and other phases in the control $(\mathrm{n}=12), \mathrm{NKH} 477(\mathrm{n}=$ $9)$, and vidarabine $(\mathrm{n}=8)$ groups $\left({ }^{+}, *, \# P<0.05\right.$ vs. phase I).

\section{Effect of parabolic flight on $L F / H F$ of $H R V$}

To further evaluate changes in autonomic nervous activity, we compared the ratio of LF to $\mathrm{HF}$ (LF/HF) in HRV analysis, an index of the sympathetic nervous tone in each phase of parabolic flight during the first parabola (16). When it was compared between normogravity (phase I) and microgravity (phase III), we found that LF/ HF was significantly lower in phase I than that in phase III in the control and NKH477 groups (Fig. 8A) [control group $(\mathrm{n}=10)$ : phase I vs. phase III: $1.9 \pm 0.4$ vs. $0.9 \pm 0.1 \mathrm{~ms}, P<0.05$; NKH477 group $(\mathrm{n}=7)$ : phase I vs. phase III, $2.1 \pm 0.04$ vs. $0.7 \pm 0.05 \mathrm{~ms}, P<$ $0.05]$. This was in agreement, at least in part, with previous studies in which decreased sympathetic nerve activity was demonstrated under microgravity in normal individuals (25). However, the vidarabine group did not show such a decrease [vidarabine group $(\mathrm{n}=8)$ : phase I vs. phase III: $1.9 \pm 0.3$ vs. $1.7 \pm 0.3 \mathrm{~ms}, P=\mathrm{NS}$, not significant], suggesting that changes in sympathetic tone be- came small when AC5 activity was decreased with vidarabine.

Under hypergravity (phase IV), the ratio of LF/HF showed a similar tendency to that under microgravity in the three groups.

When it was compared between phase I (pre-parabolic flight) and phase V (post-parabolic flight), there was no difference in control group (phase I vs. phase V: $1.9 \pm 0.4$ vs. $1.7 \pm 0.4 \mathrm{~ms}, P=\mathrm{NS}$ ) while it remained decreased in the NKH477 group (phase I vs. phase V: $2.1 \pm 0.04$ vs. $0.7 \pm 0.1 \mathrm{~ms}, P<0.05)(26)$. However, the vidarabine group showed no significant differences (phase I vs. phase V: $1.9 \pm 0.3$ vs. $1.9 \pm 0.6 \mathrm{~ms}, P=\mathrm{NS}$ ).

Accordingly, sympathetic tone was transiently decreased under micro- and hypergravity in the control while it decreased in micro- and hypergravity and remained decreased in the NKH477 group even when gravity was normalized in the post-microgravity level flight (phase V); in contrast, such decreases were all ab- 

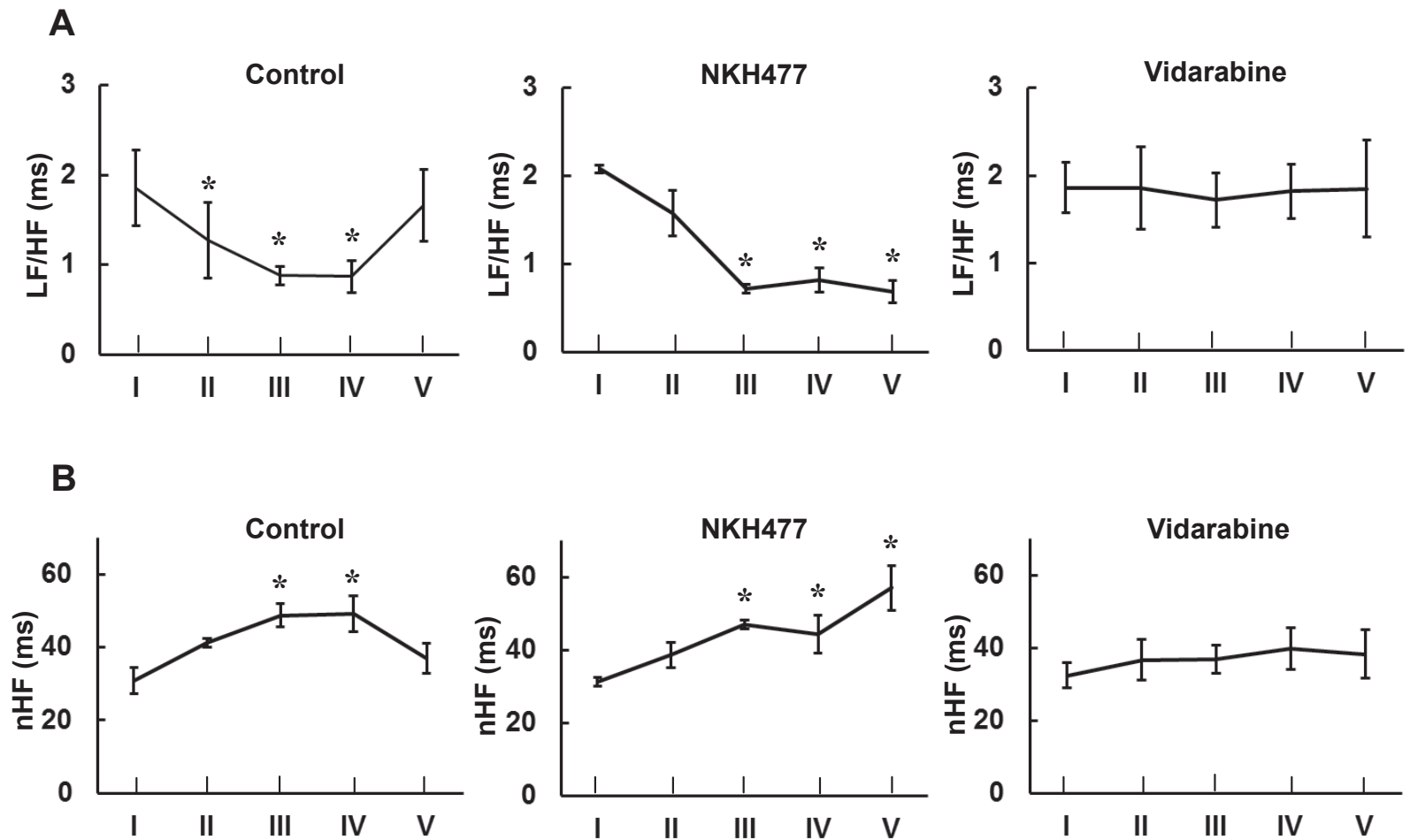

Fig. 8. Comparison of LF/HF and nHF during parabolic flights. A) LF/HF was compared as an index of sympathetic nerve activity in each phase during the first parabola in the control $(\mathrm{n}=10)$, NKH477 $(\mathrm{n}=7)$, and vidarabine $(\mathrm{n}=8)$ groups $(* P<0.05$ vs. phase I). B) nHF was compared as an index of parasympathetic nerve activity in each phase during the first parabola in control $(\mathrm{n}=10)$, NKH477 $(\mathrm{n}=7)$, and vidarabine $(\mathrm{n}=8)$ groups $(* P<0.05$ vs. phase $\mathrm{I})$.

sent in the vidarabine group.

\section{Effect of parabolic flight on $n H F$ of $H R V$}

HF power was normalized to account for differences in TP and was examined as an index of parasympathetic tone (16). When nHF was compared between normogravity (phase I) and microgravity (phase III), we found that it was significantly increased in microgravity in both the control and NKH477 groups (Fig. 8B) [control group ( $\mathrm{n}=10$ ): phase I vs. phase III: $31 \pm 3.6$ vs. $49 \pm 3.2 \mathrm{~ms}$, $P<0.05$; NKH477 group $(\mathrm{n}=7)$ : phase I vs. phase III: $31 \pm 1.2$ vs. $47 \pm 1.2 \mathrm{~ms}, P<0.05)$, suggesting that parasympathetic nerve tone was increased under microgravity in both the control and NKH477 groups. In contrast, there was no increase in $\mathrm{nHF}$ in the vidarabine group under microgravity (phase I vs. phase III: $32 \pm 3.5$ vs. $37 \pm 3.8 \mathrm{~ms}, P=\mathrm{NS}, \mathrm{n}=8$ ), suggesting that microgravity-induced enhancement of parasympathetic nerve tone was attenuated in the vidarabine group.

Under hypergravity (phase IV), nHF showed a similar tendency to that under microgravity in the three groups.

$\mathrm{nHF}$ in post-microgravity (phase V) was returned to a similar level to that in pre-microgravity (phase I) in WT $(37 \pm 4.1 \mathrm{~ms}, P=\mathrm{NS})$, while it further increased in the
NKH477 group $(57 \pm 6.2 \mathrm{~ms}, P<0.05)$ (Fig. 8B). However, there were no such increases or decreases in the vidarabine group $(38 \pm 6.6 \mathrm{~ms}, P=\mathrm{NS})$.

Thus, parasympathetic nervous tone was transiently elevated in the control group, remained elevated in the NKH477 group, and showed no changes in the vidarabine group.

Taking these results together, in comparison to the control group, the NKH477 group showed continuous responses in the above autonomic (sympathetic and parasympathetic) parameters while the vidarabine group showed attenuated responses under micro- and hypergravity.

\section{Discussion}

Our current study demonstrated that activation of AC5 activity with NKH477 could stabilize HRV, while inhibition of AC5 with vidarabine could destabilize HRV under micro- and hypergravity during parabolic flight. Further, the absolute value of the total autonomic variability index (SDNN) was much greater in the vidarabine group than that in the control or NKH477 groups under microgravity as well as hypergravity. A marker of the sympathetic 
nervous tone $(\mathrm{LF} / \mathrm{HF})$ was decreased to a greater degree in the NKH477 group relative to the control group, and a marker of parasympathetic tone (nHF) was instead increased to a greater degree in the NKH477 group; these changes were absent in the vidarabine group, indicating that pharmacological modulation of AC5 activity might be able to regulate the autonomic control of the heart rate under gravitational stress during parabolic flight.

AC consists of 9 mammalian transmembrane isoforms that differ in tissue distribution $(3,5)$, with type $6 \mathrm{AC}$ (AC6) being the major fetal cardiac $\mathrm{AC}$ isoform, and AC5, the major cardiac isoform in adults $(27,28)$. All isoforms are stimulated by Gs, but AC5 and AC6 are the only $\mathrm{Gi}$ - and $\mathrm{Ca}^{2+}$-inhibitable $\mathrm{AC}$ isoforms $(3,5)$. AC5 is responsible for at least one-third of the cardiac AC catalytic activity and plays an important role not only in sympathetic, but also parasympathetic cardiac function and heart rate without affecting Gi-gated $\mathrm{K}^{+}$-channel current, which regulates normal pacing activity, as shown recently by us and others in $\operatorname{AC5KO}(7,29)$. Overexpressing AC6 results in maintained basal HR (30), while overexpressing AC5 results in increased basal HR (13, 31 ), suggesting that AC5 may play an important role in regulating $\mathrm{HR}$ through the parasympathetic arm of the autonomic nervous system, as well as the sympathetic arm.

For many years, it has been recognized that many astronauts suffer from symptoms suggestive of autonomic dysfunction such as nausea, vomiting, and dizziness during the early days of space missions and their cardiovascular system subsequently adapts to the microgravity environment of space appropriately and effectively. However, on return to earth, from one-quarter to twothirds of them have reduced orthostatic tolerance for a few weeks. The underlying molecular mechanisms remain unknown and are probably multifactorial, although alterations in the autonomic nervous system and/or neuromuscular function after spaceflight probably contribute to this problem $(18,19)$.

We have previously demonstrated that AC5 plays an important role in stabilizing HRV under microgravity, as well as hypergravity, during parabolic flight using genetically engineered mouse models (13). This present study has demonstrated that pharmacological activation/ inhibition of AC5 activity can stabilize/destabilize the HRV not only under microgravity but also under hypergravity during parabolic flight. Importantly, NKH477 which was used in this study as a selective AC5 activator (NKH477) has been widely used for the treatment of acute heart failure in Japan since 1999. We will not need to wait for many years to get authorization for the use of NKH477 to treat autonomic dysfunction during and after space flight, if its usefulness is sufficiently confirmed by further studies.

There are plans for suborbital spaceflight or lunar tours for normal subjects as tourists, and also for prolonged space flight to Mars. It is also likely that not only astronauts, but also normal subjects will stay for up to several months in the international space station. Therefore, it is important to clarify the mechanism of the alterations in the autonomic nervous system and to develop suitable treatment. Our studies indicate that short-term, not longterm, pharmacological activation/inhibition of AC5 activity with NKH477 or vidarabine might rescue the autonomic dysfunction in the heart under microgravity in space and on earth after space flight, especially for untrained normal subjects.

\section{Acknowledgments}

This study was supported in part by grants from the Ministry of Health, Labor, and Welfare (Y.I.); the Kitsuen Kagaku Research Foundation (Y.I.); the Japanese Ministry of Education, Culture, Sports, Science, and Technology (Y.I., S.O., M.S., U.Y., R.K., Y.B., T.T., T.F.,Y.I.); Grant-in-Aid for Scientific Research on Innovative Areas (Y.I.); the Japan Space Forum (Y.I.); Takeda Science Foundation (Y.I., S.O., U.Y., M.S.); Yokohama Foundation for Advancement of Medical Science (S.O., U.Y., M.S., T.T., R.K.); Mitsubishi Pharma Research Foundation (S.O., M.S.); and Research for Promoting Technological Seeds A (discovery type) (S.O.); Yokohama Academic Foundation (S.O.); 2010 Commercialization Promotion Program for Biotechnology-related Studies (S.O.); Grant for Research and Development Project II (No. 8 and 14) of Yokohama City University (Y.I., S.O.); and Research Foundation for Community Medicine (S.O.).

\section{References}

1 Murray KJ, Reeves ML, England PJ. Protein phosphorylation and compartments of cyclic AMP in the control of cardiac contraction. Mol Cell Biochem. 1989;89:175-179.

2 Federman AD, Conklin BR, Schrader KA, Reed RR, Bourne HR. Hormonal stimulation of adenylyl cyclase through Gi-protein $\beta \gamma$ subunits. Nature. 1992;356:159-161.

3 Ishikawa Y, Homcy CJ. The adenylyl cyclases as integrators of transmembrane signal transduction. Circ Res. 1997;80:297-304.

4 Ishikawa Y. Regulation of cAMP signaling by phosphorylation. Adv Second Messenger Phosphoprotein Res. 1998;32:99-120.

5 Hanoune J, Defer N. Regulation and role of adenylyl cyclase isoforms. Annu Rev Pharmacol Toxicol. 2001;41:145-174.

6 Caulfield MP, Robbins J, Higashida H, Brown DA. Postsynaptic actions of acetylcholine: the coupling of muscarinic receptor subtypes to neuronal ion channels. Prog Brain Res. 1993;98: 293-301.

7 Okumura S, Kawabe J, Yatani A, Takagi G, Lee MC, Hong C, et al. Type 5 adenylyl cyclase disruption alters not only sympathetic but also parasympathetic and calcium-mediated cardiac regulation. Circ Res. 2003;93:364-371.

8 Okumura S, Takagi G, Kawabe J, Yang G, Lee MC, Hong C, et al. Disruption of type 5 adenylyl cyclase gene preserves cardiac function against pressure overload. Proc Natl Acad Sci 
U S A. 2003;100:9986-9990.

9 Okumura S, Vatner DE, Kurotani R, Bai Y, Gao S, Yuan Z, et al. Disruption of type 5 adenylyl cyclase enhances desensitization of cyclic adenosine monophosphate signal and increases Akt signal with chronic catecholamine stress. Circulation. 2007;116:17761783.

10 Di Rienzo M, Castiglioni P, Iellamo F, Volterrani M, Pagani M, Mancia G, et al. Dynamic adaptation of cardiac baroreflex sensitivity to prolonged exposure to microgravity: data from a 16-day spaceflight. J Appl Physiol. 2008;105:1569-1575.

11 Abe C, Tanaka K, Awazu C, Morita H. Impairment of vestibularmediated cardiovascular response and motor coordination in rats born and reared under hypergravity. Am J Physiol Regul Integr Comp Physiol. 2008;295:R173-R180.

12 Iwasaki K, Shiozawa T, Kamiya A, Michikami D, Hirayama K, Yajima K, et al. Hypergravity exercise against bed rest induced changes in cardiac autonomic control. Eur J Appl Physiol. 2005; 94:285-291.

13 Okumura S, Tsunematsu T, Bai Y, Qian J, Ono S, Suzuki S, et al. Type 5 adenylyl cyclase plays a major role in stabilizing heart rate in response to microgravity induced by parabolic flight. $\mathrm{J}$ Appl Physiol. 2008;105:173-179.

14 Toya Y, Schwencke C, Ishikawa Y. Forskolin derivatives with increased selectivity for cardiac adenylyl cyclase. J Mol Cell Cardiol. 1998;30:97-108.

15 Iwatsubo K, Minamisawa S, Tsunematsu T, Nakagome M, Toya Y, Tomlinson JE, et al. Direct inhibition of type 5 adenylyl cyclase prevents myocardial apoptosis without functional deterioration. J Biol Chem. 2004;279:40938-40945.

16 Malik M, Bigger JT, Camm AJ, Kleiger RE, Malliani A, Moss AJ, et al; Task force of the European society of cardiology and the north American society of pacing and electrophysiology. Heart rate variability. Standards of measurement, physiological interpretation, and clinical use. Eur Heart J. 1996;17:354-381.

17 Young LR, Oman CM, Watt DG, Money KE, Lichtenberg BK. Spatial orientation in weightlessness and readaptation to earth's gravity. Science. 1984;225:205-208.

18 Buckey JC Jr, Lane LD, Levine BD, Waterpaugh DE, Wright SJ, Moore WE, et al. Orthostatic intolerance after spaceflight. J Appl Physiol. 1996;81:7-18.

19 Fritsch-Yelle JM, Whitson PA, Bondar RL, Brown TE. Subnormal norepinephrine release relates to presyncope in astronauts after spaceflight. J Appl Physiol. 1996;81:2134-2141.

20 Seps B, Beckers F, Aubert AE. Heart rate variability during grav- ity transitions. Comput Cardiol. 2002;29:433-436.

21 Gehrmann J, Berul CI. Cardiac electrophysiology in genetically engineered mice. J Cardiovasc Electrophysiol. 2000;11:354368.

22 Tepe NM, Liggett SB. Transgenic replacement of type V adenylyl cyclase identifies a critical mechanism of $\beta$-adrenergic receptor dysfunction in the G $\alpha \mathrm{q}$ overexpressing mouse. FEBS Lett. 1999; 458:236-240.

23 Levine BD, Pawelczyk JA, Ertl AC, Coz JF, Zuckermann JH, Diedrich A, et al. Human muscle sympathetic neural and haemodynamic responses to tilt following spaceflight. J Physiol. 2002; 538:331-340.

24 Kamen PW, Krum H, Tonkin AM. Poincare plot of heart rate variability allows quantitative display of parasympathetic nervous activity in humans. Clin Sci (Lond). 1996;91:201-208.

25 Migeotte PF, Prisk GK, Paiva M. Microgravity alters respiratory sinus arrhythmia and short-term heart rate variability in humans. Am J Physiol Heart Circ Physiol. 2003;284:H1995-H2006.

26 Verheyden B, Beckers F, Aubert AE. Spectral characteristics of heart rate fluctuations during parabolic flight. Eur J Appl Physiol. 2005;95:557-568.

27 Tobise K, Ishikawa Y, Holmer SR, Im MJ, Newell JB, Yoshine $\mathrm{H}$, et al. Changes in type VI adenylyl cyclase isoform expression correlate with a decreased capacity for cAMP generation in the aging ventricle. Circ Res. 1994;74:596-603.

28 Espinasse I, Iourgenko V, Defer N, Salmon F, Hanoune J, Mercadier JJ. Type V, but not type VI, adenylyl cyclase mRNA accumulates in the rat heart during ontogenic development. Correlation with increased global adenylyl cyclase activity. J Mol Cell Cardiol. 1995;27:1789-1795.

29 Tang T, Lai NC, Roth DM, Drumm J, Guo T, Lee KW, et al. Adenylyl cyclase type $\mathrm{V}$ deletion increases basal left ventricular function and reduces left ventricular contractile responsiveness to $\beta$-adrenergic stimulation. Basic Res Cardiol. 2006;101: $117-126$.

30 Gao MH, Lai NC, Roth DM, Zhou J, Zhu J, Anza T, et al. Adenylylcyclase increases responsiveness to catecholamine stimulation in transgenic mice. Circulation. 1999;99:1618-1622.

31 Tepe NM, Lorenz JN, Yatani A, Dash R, Kranias EG, Dorn GW II, et al. Altering the receptor-effector ratio by transgenic overexpression of type $\mathrm{V}$ adenylyl cyclase: enhanced basal catalytic activity and function without increased cardiomyocyte $\beta$-adrenergic signalling. Biochemistry. 1999;38:16706-16713. 